\title{
Fabrication of superhydrophobic AAO-Ag multilayer mimicking dragonfly wings
}

\author{
WANG XiaoJun ${ }^{1,2}$, SONG Wei ${ }^{3}$, LI ZhiShi ${ }^{3} \&$ CONG Qian $^{1 *}$ \\ ${ }^{1}$ Key Laboratory for Bionic Engineering of Ministry of Education, Jilin University, Changchun 130025, China; \\ ${ }^{2}$ School of Chemistry and Life Science, Changchun University of Technology, Changchun 130012, China; \\ ${ }^{3}$ Key Laboratory of Supramolecular Structure and Materials of Ministry of Education, Jilin University, Changchun 130012, China
}

Received February 20, 2012; accepted May 7, 2012

\begin{abstract}
Inspired by the co-coupling of the non-smooth structure and the waxy layer inducing the hydrophobicity of dragonfly wing surface, we developed a simple and versatile method to fabricate a superhydrophobic surface with the dragonfly wing structures. In this work, Ag nanorods grew on highly ordered anodic aluminum oxide (AAO) surface via a galvanic reduction approach. Then the AAO-Ag multilayer was fabricated. Furthermore, the surface free energy of AAO-Ag multilayer was reduced by modifying with perfluorodecanethiol. The modified AAO-Ag multilayer was superhydrophobic and the static contact angle reached as high as $168^{\circ}$. X-ray photoelectron spectra (XPS) were used to characterize the chemical structure of the obtained products. The morphologies of AAO-Ag multilayer was similar to microstructure of dragonfly wing surface and presented hierarchical rough structure. The results showed that the co-coupling of the rough structure and low surface free energy induced the superhydrophobic performance of the AAO-Ag multilayer surface.
\end{abstract}

dragonfly wing, Ag, AAO, superhydrophobic, mimic

Citation: Wang X J, Song W, Li Z S, et al. Fabrication of superhydrophobic AAO-Ag multilayer mimicking dragonfly wings. Chin Sci Bull, 2012, 57: 4635-4640, doi: $10.1007 / \mathrm{s} 11434-012-5348-\mathrm{z}$

The biological microstructures are essential to remarkable hydrophobic surface for many biological system in nature [1]. Furthermore, the hierarchical roughness of microstructures optimizes superhydrophobicity on biological surfaces $[2,3]$. For example, the microrelief of plant surfaces, mainly caused by epicuticular wax crystalloids, often causes effective water repellency [4]. The rough, waxy leaves of plants are not only water-repellent but anti-adhesive with respect to particulate contamination. The contaminating particles are carried away by water droplets, resulting in a cleaned surface (Lotus-effect) [5]. Hierarchical micropapillae and nanofolds that exist on the petals' surfaces of red roses provide a sufficient roughness for superhydrophobicity and yet at the same time a high adhesive force with water [6]. Moreover, It was found that some insects with very unwettable wings show a highly significant "self- cleaning" effect

*Corresponding author (email: congqian@jlu.edu.cn) under the influence of rain or dew [7]. The hierarchical multiple roughness structures on the wing surfaces of insects enhanced their hydrophobicity [8]. The multivariate coupling of the shape, structures and biomaterial of the scales induced the hydrophobicity of the butterfly and moth wings $[9,10]$. The cooperation of nanogroove structures on the oriented microsetae on water strider's leg, in conjunction with the wax on the leg, renders such water repellency $[11,12]$. Inspired by these, many artificial surfaces with special wettability have been prepared in a variety of ways, including template method [13], sol-gel processing [14], self-assembly method $[15,16]$, electrochemical deposition [17], electrospinning method [18], electroless galvanic deposition method [19], chemical etching [20], chemical vapor deposition method(CVD) [21], and so on [22-30].

Moreover, it was found that the contact angle on dragonfly (pantala flavescens fabricius) wing surface was about $146^{\circ}$ [31]. In addition, the membrane of dragonfly wing in the di- 
rection of the thickness was divided into three layers, which were separately referred to as dorsal surface, middle layer and ventral surface. And both the dorsal and the ventral surfaces displayed a random distributed rough microstructure that was composed of numerous nanometer scale columns coated by the cuticle wax secreted [32,33]. The hydrophobicity of dragonfly wing surface was induced by the co-coupling of the non-smooth structure and the waxy layer [31].

Inspired by dragonfly wing surface, we developed a simple and versatile method to fabricate a superhydrophobic surface. First, the highly ordered anodicaluminum oxide (AAO) has been fabricated using a two-step anodization approach. Secondly, the as-synthesized AAO film was used as substrate and $\mathrm{Ag}$ nanorods grew on the substrate via a galvanic reduction. Then the AAO-Ag multilayer was fabricated. Finally, the AAO-Ag multilayer film would be modified by hydrophobic substance in order to achieve a superhydrophobicity on its surface. So, the superhydrophobic surface was obtained mimicking dragonfly wings. It will give us more experiences and design principles to construct bionic and superhydrophobic surface.

\section{Experimental}

\subsection{Chemicals and materials}

1H,1H,2H,2H-perfluorodecanethiol (PDT) was purchased from Sigma-Aldrich Co. (UK). All the other reagents used were of analytical reagents (AR) grade without further purification and was obtained from the Beijing Chem. Co. (China). The water used was purified through a distillation system.

\subsection{Preparation of anodized aluminum oxide (AAO)}

Aluminum foils $(99.999 \%$, General Research Institute for Nonferrous Metals, China) were annealed at $500^{\circ} \mathrm{C}$ for $2 \mathrm{~h}$ in nitrogen atmosphere and were cut into $1 \mathrm{~cm} \times 2 \mathrm{~cm}$. Because $\mathrm{Al}$ foil surfaces were anodization in air and degreased, it was necessary to be etched in sodium hydroxide solution, rinsed in distilled water and dried by nitrogen gas. In a typical procedure [34], Al foils were electropolished for $90 \mathrm{~s}$ in a perchloric acid solution (perchloric acid: absolute ethanol $=1: 4)$ at $17 \mathrm{~V}$ and room temperature, then were anodized in oxalic acid $(0.3 \mathrm{~mol} / \mathrm{L})$ at $40 \mathrm{~V}$ and $4^{\circ} \mathrm{C}$ for $4 \mathrm{~h}$ with stirring. The first grown oxide layers were then etched out completely with a chromic acid solution (1.5 wt $\%$ chromic acid and $6 \mathrm{wt} \%$ phosphoric acid in DI water)at $60^{\circ} \mathrm{C}$, and a well-ordered porous AAO was grown by the second anodization for $4 \mathrm{~h}$ [35].

\subsection{Ag nanorods growing on AAO surface via a galvanic reduction approach}

The as-prepared AAO over aluminum foil was immersed into the ammoniate silver solution $(0.1 \mathrm{~mol} / \mathrm{L})$ for $10 \mathrm{~min}$ at room temperature (about $298 \mathrm{~K}$ ). After that, $50 \mu \mathrm{L}$ of formaldehyde reducing agent $(4 \mathrm{~mL}$ formaldehyde, $4 \mathrm{~mL}$ deionized water and $92 \mathrm{~mL}$ absolute ethanol were mixed) was dripped into the above ammoniate silver solution drop by drop. After 15 min the sample was taken out from the ammoniate silver solution, rinsed with a large amount of deionized water $(50 \mathrm{~mL})$ for three times, then dried under a flow of nitrogen gas at room temperature. The schematic diagram of the preparation procedure for AAO-Ag multilayer was showed in Figure 1.

\subsection{AAO-Ag multilayer film modified by PDT}

Both AAO over aluminum foil and as-prepared Ag-AAO multilayer nanostructures were immersed into ethanol solution of low surface energy agent PDT for 3 days, then rinsed with deionized water, and dried under a flow of nitrogen gas.

\subsection{Characterization and measurement}

Scanning electron microscopy (SEM) images were taken on a field-emission scanning electron microscope (JEOL, JSM-6700F, Japan) with an acceleration voltage of $3.0 \mathrm{kV}$. Samples were sputtered with a layer of gold ( 10 nm thick) prior to imaging. XPS analysis of the X-ray photoelectron spectra (XPS) of Ag nanoparticles on the tip of AAO nanorods over $\mathrm{Al}$ foil was measured by use of on an ESCLAB MKII using $\mathrm{Al}$ as the exciting source. The contact angles of $3 \mu \mathrm{L}$ water drop were measured with video-based optical contact angle measuring instruments (Dataphysics, OCA20, Germany) at ambient temperature.

\section{Results and discussion}

\subsection{Surface morphologies before and after $\mathrm{Ag}$ nanorods growing on the tip of $\mathrm{AAO}$}

Figure 2(a)-(d) showed the SEM images of aluminum foil, $\mathrm{AAO}$ and as-prepared AAO-Ag multilayers. Figure 2(a) showed the SEM image of the smooth aluminum foil. Figure 2(b) showed the SEM image of top view of AAO prepared at $40 \mathrm{~V}$. As reported previously [36], a highly ordered hexagonal arrangement of the pores was obtained under the appropriate anodization condition and the applied voltage of $40 \mathrm{~V}$ was ideal for the formation of stable porous structure. The pore diameter of as-prepared AAO is about $30 \mathrm{~nm}$. Figure 2(c) showed the SEM image of cross section view of AAO. As shown in Figure 2(c), the thickness of AAO is about $3 \mu \mathrm{m}$.

Figure 2(d) showed a SEM image of the as-prepared Ag-AAO multilayer nanostructures. The formation of these regular Ag-AAO heterogeneous nanostructures can be explained by a localization of the electrons at the ends of 


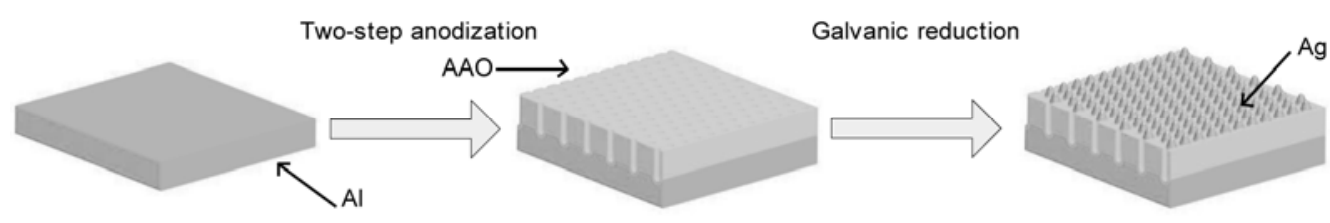

Figure 1 Schematic of the fabrication of AAO-Ag multilayer.

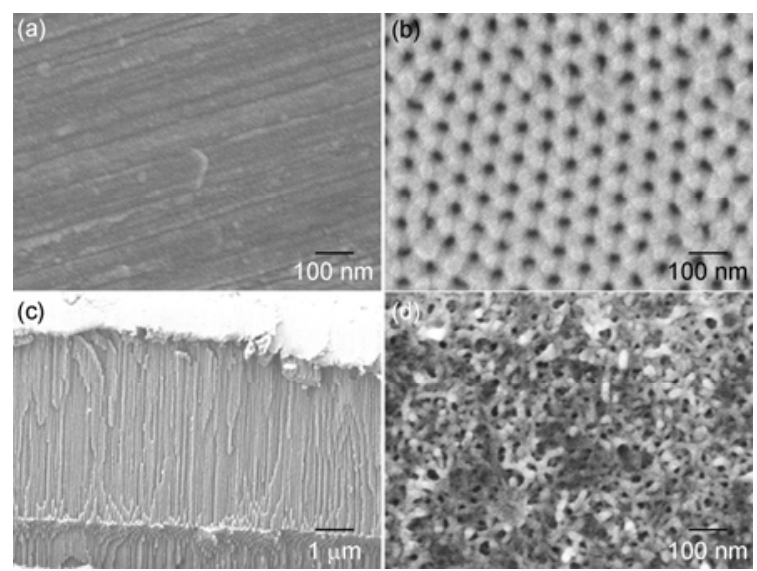

Figure 2 SEM images of the products. (a) $\mathrm{Al}$; (b) top view of AAO; (c) cross section view of AAO; (d) Ag nanorods growing on the tip of AAO.

AAO after the electron transfer step. After AAO over $\mathrm{Al}$ was immersed in ammoniate silver solution for $10 \mathrm{~min}$, the electron can move from the Al plate to the top of AAO, the electron density of the top of AAO is enormously increased and the silver ions in solution obtain an electron to produce Ag nanoparticles around the top of AAO. This results is consistent with the previous report for the fabrication of the nanoheterostructure of $\mathrm{ZnO}$ nanorod arrays with attached spherical Ag nanoparticles on their tips [37]. After formaldehyde reducing agent is added, Ag nanorods grow further on the tip of AAO taking Ag nanoparticles as seeds. The length of Ag nanorods synthesized on the surface of AAO is in the range of $50-70 \mathrm{~nm}$, and the diameter is about $20 \mathrm{~nm}$, which is clearly shown in the enlarged SEM image. The formed $\mathrm{Ag}-\mathrm{AAO}$ heterogeneous nanostructures exhibited as pile loop of towel, just like microstructure of dragonfly wing surface (pantala flavescens fabricius, showed in Figure 3(a)).

\subsection{XPS analysis}

The chemical composition of as-prepared Ag-AAO multilayer film was characterized by XPS measurements. Figure 4(a)-(e) displayed the XPS spectra of C 1s, N 1s, O 1s, Ag $3 \mathrm{~d}$ and $\mathrm{Al} 2 \mathrm{p}$ core levels for AAO-Ag multilayer film, respectively. The peaks corresponding to $\mathrm{Ag} 3 \mathrm{~d}$ were recorded in the spectrum with binding energy located at 368.5 and $374.5 \mathrm{eV}$ (Figure. 4(d)). The line shape of the core-level O $1 \mathrm{~s}$ and $\mathrm{Al} 2 \mathrm{p}$ were showed with a binding energy of 532.0 (Figure 4(c)) and $74.5 \mathrm{eV}($ Figure 4(e)), respectively. The XPS results indicated that $\mathrm{Ag}$ nanorods have covered the

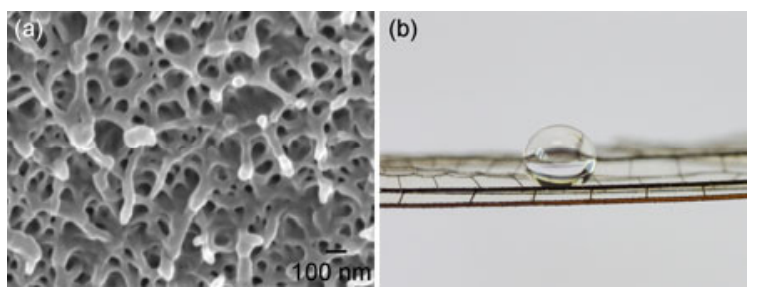

Figure 3 Dragonfly wing. (a) SEM image of dragonfly wing; (b) water droplet on dragonfly wing.

AAO membrane completely.

\subsection{Wettability properties before and after Ag nano- rods growing on the tip of AAO}

The contact angles of $3 \mu \mathrm{L}$ water droplet on the aluminum foil, AAO film and the as-prepared AAO-Ag multilayer are measured. As shown in Figure 5(a) and (b), the contact angle on natural oxide of alumina surface is $52^{\circ}$ and the contact angle on AAO surface is $35^{\circ}$. The two contact angles are both very low. However, there is great difference in surface wettability before and after $\mathrm{Ag}$ nanorods growing and modification by PDF on the tip of AAO. As seen in Figure 5(c), the contact angle on as-prepared Ag-AAO multilayer nanostructure surface modified by PDT is extraordinary great and the average value is $168^{\circ}$. The state of water droplet on AAO-Ag multilayer nanostructure surface modified can maintain perfect sphericity, which is similar to the state on the dragonfly wing surface (Figure 3(b)). So the superhydrophobic characteristic of the dragonfly wing is successfully imitated.

\subsection{Mechanism analysis of superhydrophobicity on AAO-Ag multilayer}

Typically, the solid surface with the contact angle over $150^{\circ}$ is considered as super-hydrophobic surface. At present, there are two prominent models: Wenzel state and Cassie state [38-40]. AAO is metal oxide with hydrophilic properties, and the surface structure is highly ordered and porous. When water drop contacts hydrophilic AAO surface with fine roughness structure, it will have a capillary effect, which results in water being sucked within the porous structure on AAO surface. The contact state of water droplets and rough surface is shown in Figure 6(a). So the drop wets the grooves, in this case, the Wenzel equation is applied [41].

$$
\cos \theta_{r}=r \cos \theta_{e},
$$



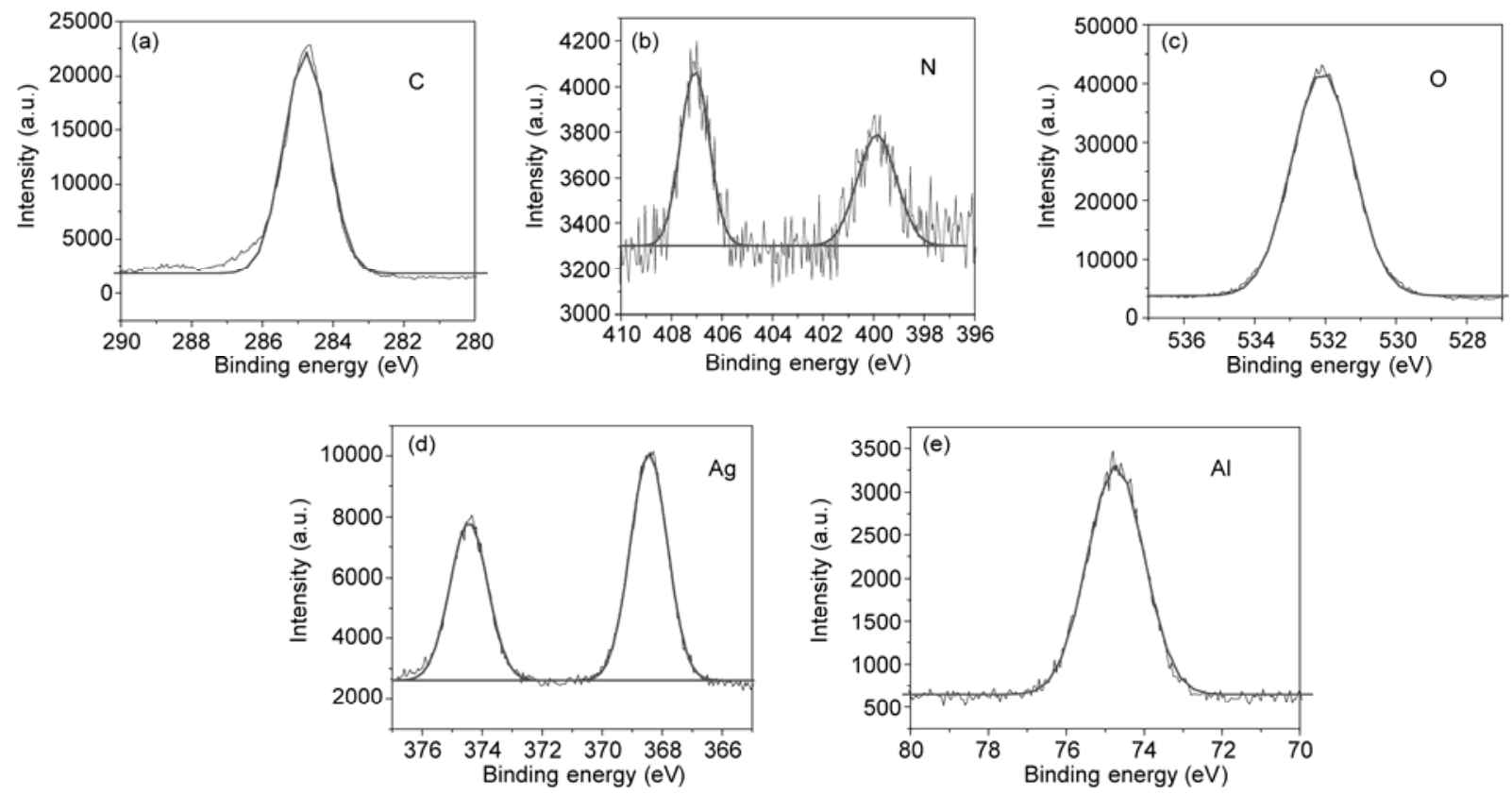

Figure 4 XPS spectra. (a) C; (b) N; (c) O; (d) Ag and (e) Al.
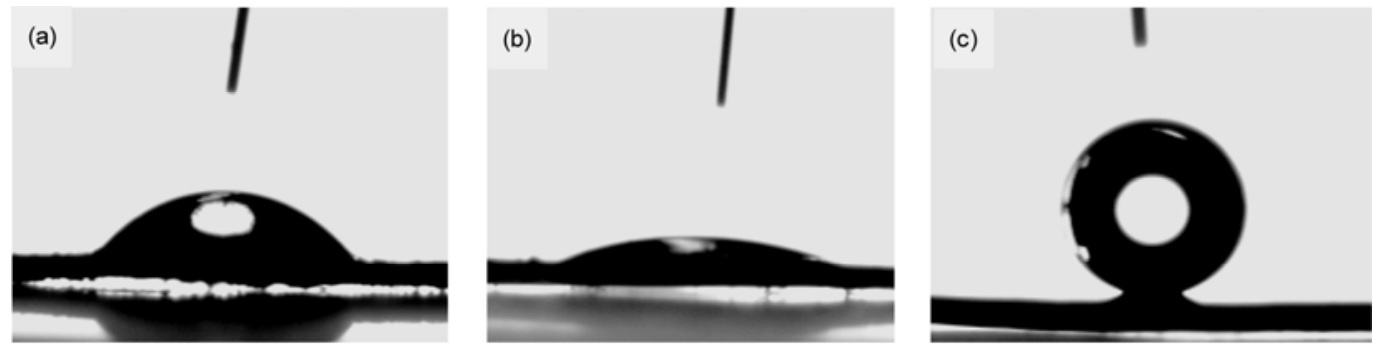

Figure 5 The CA optical image between water droplet and sample. (a) Al; (b) AAO; (c) Ag-AAO multilayer.
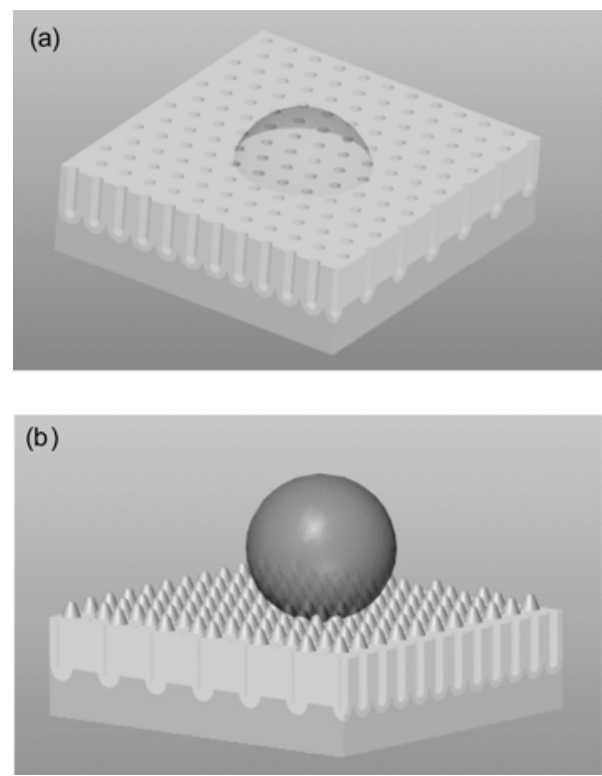

Figure 6 Two contact states (a) the contact state between water drop and $\mathrm{AAO}$; (b) the contact state between water drop and AAO-Ag multilayer film. where $r$ is defined as the roughness factor, it is the ratio of the actual solid-liquid interface contact area and the apparent solid-liquid interface contact area; $\theta_{r}$ is the apparent contact angle, and $\theta_{e}$ is the intrinsic contact. In this experiment, the measured contact angle between natural oxide of alumina surface and water droplet is $52^{\circ}$, it is considered as $\theta_{e}$, while contact angle on AAO surface $\theta_{r}$ is $35^{\circ}$. The two contact values are substituted into equation (1), then $r$ is calculated as 1.33. According to Wenzel equation, the surface roughness makes the hydrophilic surface $\left(\theta_{e}<90^{\circ}\right)$ is more hydrophilic. When roughness factor $r$ increases, the contact angle decreases.

After Ag nanorods grow on the surface of AAO, the sample surface presents multiscale and multilayer nanocomposite structure. Furthermore, the as-prepared AAO-Ag multilayer film is modified by PDT molecules through metal-thiol coordination bond to form a self-assembled monolayer with a low surface energy. For porous material or rough surface trapping the air, the contact between drop and the rough surface is compound contact. At this point, Cassie 
equation can be used. The AAO-Ag multilayer film is so sufficiently rough that the water droplet may trap air. And then the surface is made up of solid and air components so as to give a composite surface effect. So contact state between water droplet and the multilayer film surface accords with Cassie model, as shown in Figure 6(b). It is described in eq. (2) [42]:

$$
\cos \theta_{r}=r_{w} \phi_{s} \cos \theta_{e}+\phi_{s}-1
$$

where $\phi_{s}$ is the area fraction on the horizontal projected plane of the liquid-solid contact and $r_{w}$ is the ratio of the actual area to the projected area of liquid-solid contact. In this experiment, the apparent contact angle $\theta_{r}$ is $168^{\circ}$. The co-coupling effect of chemical composition and nanocomposite structure induces the super-hydrophobicity of AAO-Ag multilayer film.

\section{Conclusions}

Dragonfly wing structure are divided into three layers, which were dorsal surface, middle layer and ventral surface. And there are a large number of mastoids covered by hydrophobic waxy on the surface of dragonfly wing. The co-coupling effect of rough multilayer structure and hydrophobic waxy composition induces super-hydrophobic properties on dragonfly wing surface. Inspired by this, AAO nanostructure is prepared and $\mathrm{Ag}$ nanorods grow on the AAO surface. Thus the rough multilayer of AAO-Ag nanostructure is formed. Moreover, the AAO-Ag nanocomposite film is modified by hydrophobic PDT. It achieves ultimately the super-hydrophobic properties on AAO-Ag multilayer surface, and the static contact angle reaches $168^{\circ}$. The results show that increasing the multi-scale roughness and modification of hydrophobic substances can improve the superhydrophobic performance on solid surface. In this study, mimetic materials similar to dragonfly wings is prepared, they have excellent super-hydrophobic properties. This paper provides new ideas and methods to design and fabricate mimetic superhydrophobic materials, and that to be applied to all areas of production and life.

This work was supported by the National Natural Science Foundation of China (50875108 and 20903044), the Specialized Research Fund for the Doctoral Program of Higher Education of China (20100061110022) and the Open Project Program of Key Laboratory for Bionic Engineering of Ministry of Education.

1 Bhushan B. Biomimetics: Lessons from nature-An overview. Philos Trans R Soc A, 2009, 367: 1445-1486

2 Bhushan B, Sayer R A. Surface characterization and friction of a bio-inspired reversible adhesive tape. Microsyst Technol, 2007, 13: 71-78

3 Miwa M, Nakajima A, Fujishima A, et al. Effects of the surface roughness on sliding angles of water droplets on superhydrophobic surfaces. Langmuir, 2000, 16: 5754-5760

4 Barthlott W, Neinhuis C. Purity of the sacred lotus,or escape from contamination in biological surfaces. Planta, 1997, 202: 1-8

5 Neinhuis C, Barthlott W. Characterization and distribution of waterrepellent, self-cleaning plant surfaces. Ann Bot-London, 1997, 79: 667-677

6 Feng L, Zhang Y N, Xi J M, et al. Petal effect: A superhydrophobic state with high adhesive force. Langmuir, 2008, 24: 4114-4119

7 Wagner T, Neinhuis C, Barthlott W. Wettability and contaminability of insect wings as a function of their surface sculptures. Acta ZoolStockholm, 1996, 77: 213-225

8 Byun D, Hong J, Saputra, et al. Wetting characteristics of insect wing surfaces. J Bionic Eng, 2009, 6: 63-70

9 Fang Y, Sun G, Wag T Q, et al. Hydrophobicity mechanism of non-smooth pattern on surface of butterfly wing. Chin Sci Bull, 2007, 52: 711-716

10 Wang X J, Cong Q, Zhang J J, et al. Multivariate coupling mechanism of NOCTUIDAE moth wings' surface superhydrophobicity. Chin Sci Bull, 2009, 54: 569-575

11 Feng X Q, Gao X F, Wu Z N, et al. Superior water repellency of water strider legs with hierarchical structures: Experiments and analysis. Langmuir, 2007, 23: 4892-4896

12 Dickinson M. How to walk on water. Nature, 2003, 424: 621-622

13 Xi J M, Jiang L. Biomimic superhydrophobic surface with high adhesive forces. Ind Eng Chem Res, 2008, 47: 6354-6357

14 Pilotek S, Schmidt H K. Wettability of microstructured hydrophobic sol-gel coatings. J Sol-Gel Sci Techn, 2003, 26: 789-792

15 Kemell M, Färm E, Leskelä M, et al. Transparent superhydrophobic surfaces by self-assembly of hydrophobic monolayers on nanostructured surfaces. Phys Status Solidi A, 2006, 203: 1453-1458

16 Ji J, Fu J H, Shen J C. Fabrication of a superhydrophobic surface from the amplified exponential growth of a multilayer. Adv Mater, 2006, 18: 1441-1444

17 Li M, Zhai J, Liu H, et al. Electrochemical deposition of conductive superhydrophobic zinc oxide thin films. J Phys Chem B, 2003, 107: 9954-9957

18 Zhu Y, Zhang J, Zheng Y, et al. Stable, superhydrophobic, and conductive polyaniline/polystyrene films for corrosive environments. Adv Funct Mater, 2006, 16: 568-574

19 Xu X H, Zhang Z Z, Yang J. Fabrication of biomimetic superhydrophobic surface on engineering materials by a simple electroless galvanic deposition method. Langmuir, 2010, 26: 3654-3658

20 Qian B T, Shen Z Q. Fabrication of superhydrophobic surfaces by dislocation-selective chemical etching on aluminum, copper, and zinc substrates. Langmuir, 2005, 21: 9007-9009

21 Cremona A, Vassallo E, Merlo A, et al. Synthesis by plasmaenhanced chemical-vapor deposition and characterization of siliconlike films with hydrophobic functionalities for improved long-term geometric stability of fiber-reinforced polymers. J Mater Res, 2008, 23: 1042-1050

22 Sun T L, Wang G J, Liu H, et al. Control over the wettability of an aligned carbon nanotube film. J Am Chem Soc, 2003, 125: 1499614997

23 Fürstner R, Barthlott W, Neinhuis C, et al. Wetting and self-cleaning properties of artificial superhydrophobic surfaces. Langmuir, 2005, 21: 956-961

24 Xi J M, Jiang L. Biomimic superhydrophobic surface with high adhesive forces. Ind Eng Chem Res, 2008, 47: 6354-6357

25 Zhang H, Guo D J, Dai Z D. Progress on gecko-inspired micro/nano-adhesion arrays. Chin Sci Bull, 2010, 55: 1843-1850

26 Zhang D Y, Li Y, Han X, et al. High-precision bio-replication of synthetic drag reduction shark skin. Chin Sci Bull, 2011, 56: 938944

27 Liu L J, Zhao J S, Zhang Y, et al. Fabrication of superhydrophobic surface by hierarchical growth of lotus-leaf-like boehmite on aluminum foil. J Colloid Interf Sci, 2011, 358: 277-283

28 Kustandi T S, Low H Y, Teng J H, et al. Mimicking domino-like photonic nanostructures on butterfly wings. Small, 2009, 5: 574-578

29 Liu K S, Du J X, Wu J T, et al. Superhydrophobic gecko feet with 
high adhesive forces towards water and their bio-inspired materials. Nanoscale, 2012, 4: 768-772

30 Lee W, Park B G, Kim D H, et al. Nanostructure-dependent water-droplet adhesiveness change in superhydrophobic anodic aluminum oxide surfaces: From highly adhesive to self-cleanable. Langmuir, 2010, 26: 1412-1415

31 Wan Y L, Cong Q, Wang X J, et al. The wettability and mechanism of geometric non-smooth structure of dragonfly wing surface. J Bionic Eng, 2008, 5(Suppl): 40-45

32 Song F, Lee K L, Soh A K, et al. Experimental studies of the material properties of the forewing of cicada (Homóptera, Cicàdidae). J Exp Biol, 2004, 207: 3035-3042

33 Song F, Xiao K W, Baib K, et al. Microstructure and nanomechanical properties of the wing membrane of dragonfly. Mat Sci Eng A-Struct, 2007, 457: 254-260

34 Lu Z C, Ruan W D, Yang J X, et al. Deposition of Ag nanoparticles on porous anodic alumina for surface enhanced Raman scattering substrate. J Raman Spectrosc, 2009, 40: 112-116
35 Jessensky O, Muller F, Gosele U. Self-organized formation of fexagonal pore arrays in anodic alumina. Appl Phys Lett, 1998, 72: 1173-1175

36 Zhao Y C, Chen M, Zhang Y N, et al. A facile approach to formation of through-hole porous anodic aluminum oxide film. Mater Lett, 2005, 59: 40-43

37 Song W, Han X X, Chen L, et al. Site-specific deposition of Ag nanoparticles on $\mathrm{ZnO}$ nanorod arrays via galvanic reduction and their SERS applications. J Raman Spectrosc, 2009, 41: 907-913

38 Patankar N A. Transition between superhydrophobic states on rough surfaces. Langmuir, 2004, 20: 7097-7102

39 Cassie A B D, Baxter S. Wettability of porous surfaces. Trans Faraday Soc, 1944, 40: 546-551

40 Wenzel R N. Resistance of solid surfaces to wetting by water. J Ind Eng Chem, 1936, 28: 988-994

41 Wenzel R N. Surface roughness and contact angle. J Phys Chem, 1949, 53: 1466-1467

42 Cassie A B D. Contact angles. Discuss Faraday Soc, 1948, 3: 11-16

Open Access This article is distributed under the terms of the Creative Commons Attribution License which permits any use, distribution, and reproduction in any medium, provided the original author(s) and source are credited. 